\title{
AVALIAÇÃO DA REPELÊNCIA DE ALLIUM SATIVUM, L. (ALHO) VISANDO O CONTROLE DE NAUPHOETA CINEREA (OLIVIER, 1789)
}

\section{REPELLENCY EVALUATION OF ALLIUM SATIVUM, L. (GARLIC) FOR THE CONTROL OF NAUPHOETA CINEREA (OLIVIER, 1789)}

\author{
Anna Clara Balbina Silva ${ }^{1}$ \\ Afonso Pelli ${ }^{2}$
}

Submetido: 30/03/2021 / Aprovado: 28/10/2021 / Publicado: 24/11/2021.

\begin{abstract}
Resumo
Alguns dos princípios ativos provenientes de plantas podem causar diferentes efeitos sobre os insetos como repelência. Dentre as plantas utilizadas encontra-se o alho. Seu odor característico está relacionado com a presença de metabólitos secundários, que eventualmente podem proteger a planta contra insetos e fitopatógenos. Objetivou-se com este trabalho avaliar a ação repelente de Allium sativum L. (alho), sobre Nauphoeta cinerea (Olivier, 1789) (Blattodea: Blaberidae). Foram utilizados três indivíduos, um sem marcação, e dois marcados com corretivo líquido à base de água (New Magic $($ ), um desses com tinta azul e o outro com tinta vermelha, foram mantidos em uma caixa plástica, cuja tampa foi retirada e colocada um sombrite, o fundo da caixa foi dividido em quatro quadrantes numerados, com alimento e água disponível, e acompanhados por um período de duas horas, com o extrato vegetal colocado em um dos quadrantes. Os dados apontaram eficácia com o uso do extrato aquoso, do óleo Guarlic Guard®, do extrato alcoólico e do vegetal a seco. O vegetal in natura não teve resultado significativo. Sendo assim, extratos vegetais de A. sativum podem ser uma alternativa a inseticidas químicos no controle de populações de baratas, apresentando vantagens de baixo custo econômico, de fácil preparo e com menor impacto ambiental.
\end{abstract}

Palavras-chave: Alicina. Blaberidae. Insetífugo. Composto químico.

\begin{abstract}
Some of the active ingredients from plants can cause different effects on insects such as repellency. Among the plants used is garlic. Its characteristic odor is related to the presence of secondary metabolites, which may eventually protect the plant against insects and phytopathogens. The objective of this work was to evaluate the repellent action of Allium sativum L. (garlic), on Nauphoeta cinerea (Olivier, 1789) (Blattodea: Blaberidae). Three individuals were used, one unmarked, and two marked with water-based liquid concealer (New Magic ${ }^{\circledR}$ ), one with blue paint

\footnotetext{
${ }^{1}$ Mestre em Ciência e Tecnologia ambiental. Departamento de Patologia, Genética e Evolução. Instituto de Ciências Biológicas e Naturais. Universidade Federal do Triângulo Mineiro. Av. Frei Paulino, 30. Uberaba/MG, Brasil. CEP 38025-180. E-mail: annaclara1996@live.com

${ }^{2}$ Doutor em Aquicultura. Departamento de Patologia, Genética e Evolução. Instituto de Ciências Biológicas e Naturais. Universidade Federal do Triângulo Mineiro. Av. Frei Paulino, 30. Uberaba/MG, Brasil. CEP 38025-180. Email:apelli@terra.com.br
} 
and the other with red paint, kept in a plastic box, whose lid was removed and placed a shade, the bottom of the box was divided into four numbered quadrants, with food and water available, and followed for a period of two hours, with the plant extract placed in one of the quadrants. The data showed effectiveness with the use of aqueous extract, Guarlic Guard ${ }^{\circledR}$ oil, alcoholic extract and dry vegetable. The fresh vegetable had no significant result. Thus, plant extracts of A. sativum can be an alternative to chemical insecticides in the control of cockroach populations, presenting advantages of low economic cost, easy to prepare and with less environmental impact.

Keywords: Allicin. Blaberidae. Insectifugal. Chemical compost.

\section{INTRODUÇÃo}

Com o aumento dos centros urbanos e das populações, observa-se o surgimento de ambientes e hábitos que favorecem o crescimento de pragas, como as baratas. Estas se alimentam de restos orgânicos gerados pela população, gerando assim a necessidade de métodos de controle. Os métodos comumente usados para o controle de baratas são baseados no uso de produtos químicos e instalações de barreiras físicas (SANTOS et al., 2019).

Entretanto, devido ao aumento da resistência aos inseticidas utilizados, algumas plantas incluindo as medicinas têm sido estudadas como alternativas para prevenção e controle de insetos. (MELO et al.,2011). Alguns dos princípios ativos provenientes de plantas podem causar diferentes efeitos sobre os insetos como repelência, inibição da alimentação, da reprodução e mesmo mortalidade nas diversas fases da vida (ADIRANO-ANAYA et al., 2018).

Neste contexto, diversos estudos relacionados ao uso dos extratos vegetais têm sido desenvolvidos, uma diversidade de compostos de plantas já foram isolados e separada com intuito de medidas de controle para insetos. Dentre as plantas utilizadas encontra-se o Allium sativum L., que pertence à família Alliaceae. Seu cultivo é cosmopolita e existe grande demanda pelas suas propriedades medicinais (FONSECA et al., 2014) e uso na culinária tradicional (OLIVEIRA et al., 2014).

As altas quantidades de amido e compostos aromáticos participam da composição do alho o que significa que possui um alto valor medicinal e sabor peculiar. Também é utilizado como fitoterápico devido às suas diversas propriedades farmacológicas (CAVALCANTI et al., 2018). O seu forte odor está relacionado com a presença de metabólitos secundários, como compostos organosulfurados, que protegem a planta contra insetos e fitopatógenos (KUSANO et al., 2016). O componente responsável pelas características antibiótica, antiviral e antifúngica da planta é a Alicina (BORLINGHAUS et al., 2014).

Alguns insetos como as baratas são vetores mecânicos de vários microrganismos, podendo assim carregar vírus, bactérias, fungos e protozoários, eventualmente patogênicos. Desta forma apresentam grande importância, como vetores mecânicos de agentes etiológicos como hepatite A, febre tifoide e diarreias (KAKUMANU et al., 2018), por causarem prejuízos a produtos armazenados e também atuando como hospedeiros intermediários de muitos helmintos do homem e animais domésticos. Além disso, por terem alto potencial reprodutivo e capacidade de se adaptar em diferentes ambientes possivelmente podem se tornar um problema para o equilíbrio ambiental (SILVA et al., 2019).

A barata Nauphoeta cinerea (Olivier, 1789) é da família Blaberidae, oriunda do Leste da África, sua atual distribuição se encontra nos trópicos. Vivem aproximadamente 1 ano, e realizam entre 8 ecdises em média nesse período. Os adultos conseguem atingir entre $25-29 \mathrm{~mm}$ de comprimento, suas asas são manchadas com cor acinzentada e não cobrem todo o abdômen. São onívoras, e aceitam tanto alimento animal quanto vegetal (SANTOS et al., 2019). Essa espécie tem sido utilizada como enriquecimento alimentar na ração de ruminantes, pelo alto teor de proteína e 
o baixo custo de produção, sendo encontrada em todo território nacional brasileiro, também utilizadas em substituição de mamíferos como modelos para estudos toxicológicos e como alimentos para outros insetos em biotérios (SILVA et al., 2020). Neste contexto, este trabalho teve como objetivo testar a eficiência da repelência do alho sobre Nauphoeta cinerea.

\section{MATERIAL E MÉTODOS}

O experimento foi conduzido no Laboratório de Ecologia \& Evolução Nico Nieser do Departamento de Patologia, Genética e Evolução da Universidade Federal do Triângulo Mineiro.

Os animais foram criados e mantidos em biotério, com controle de fotoperíodo, 12 horas claro/escuro utilizando timer temporizador, a temperatura permanece abaixo de $26-27^{\circ} \mathrm{C}$, e sem controle da umidade, em setembro de 2019, na cidade de Uberaba/MG. A licença para coleta de insetos foi concedida pelo ICBMBIO, sob $\mathrm{n}^{\circ}$ 63276-1, sendo o biotério registrado na Pró-Reitoria de Pesquisa e Pós-Graduação da Universidade Federal do Triângulo Mineiro e no Conselho Regional de Biologia $4^{\mathrm{a}} \mathrm{Rg}$, sob responsabilidade e Anotação de Responsabilidade Técnica do biólogo.

Os animais são criados e mantidos em caixa plástica com 30 × 20 x $15 \mathrm{~cm}$, com cama de maravalha de eucalipto. Nas bordas utilizou-se vaselina sólida para evitar fugas. As baratas são alimentadas uma vez por semana com ração para peixes $55 \%$ de proteína bruta da Guabi® (SILVA, PELLI, 2020) para alevinos.

Foram utilizados três indivíduos, um sem marcação, e dois marcados com corretivo líquido à base de água (New Magic $®)$, um desses com tinta azul e o outro com tinta vermelha. Estes foram adquiridos de matrizes já existentes no laboratório. O indivíduo Um ficou sem marcação, o indivíduo Dois foi marcado na parte posterior do corpo, nos tergos abdominais 7 a 10, com a cor vermelha e o indivíduo Três marcado no mesmo local com a cor azul. Estes foram mantidos em sala com a temperatura entre $26-27^{\circ} \mathrm{C}$, em caixa plástica com $30 \times 20 \times 15 \mathrm{~cm}$, com pouca serragem no fundo, sendo a tampa substituída por um sombrite transparente, possibilitando a visualização dos indivíduos.

No fundo da caixa foram demarcados quatro quadrantes numerados, cada um dos quadrantes possuía um recipiente com água (Figura 1). O alimento utilizado foi ração de peixe da Guabi® 55\% de proteína bruta, disposta sobre a serragem. A caixa foi observada uma vez a cada quinze minutos, por um período de duas horas; e anotado o número do quadrante no qual o indivíduo estava. Quando o indivíduo se encontrava na divisão de dois quadrantes, era considerado o quadrante em que a cabeça estava. 


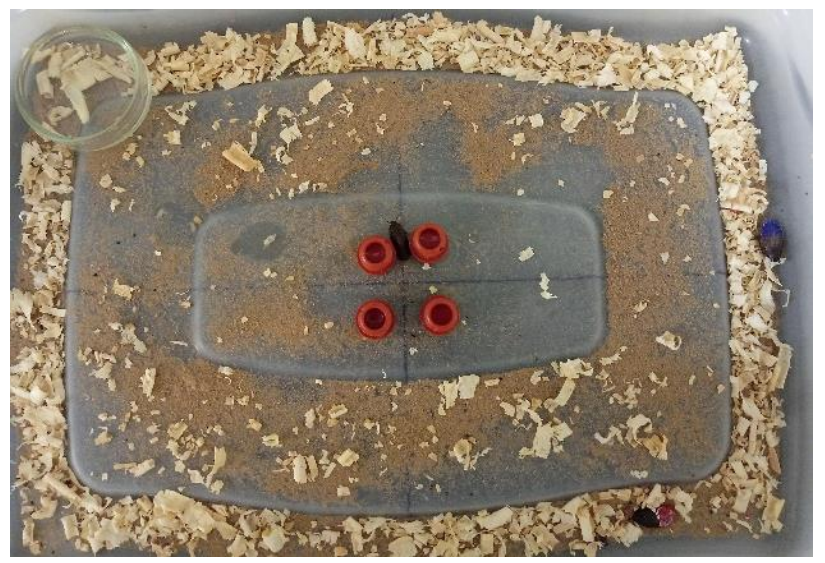

Figura 1. Caixa de experimentação utilizada durante observação dos testes de repelência de Nauphoeta cinerea (Olivier, 1789) em condições não controladas de laboratório.

A espécie vegetal, utilizada para obtenção dos extratos vegetais, foi adquirida no município de Uberaba, localizado a $477 \mathrm{~km}$ de Belo Horizonte, localização geodésica aproximada 1944'60', S e 47054'33' W. O material vegetal foi pesado em balança Shimadzu, modelo AX200 e dividido em quatro amostras de $300 \mathrm{mg}$; sendo acondicionado separadamente em tubos de vidro medindo $15 \times 150 \mathrm{~mm}$, com tampa rosqueada e capacidade para $14 \mathrm{~mL}$ por um período de 72 horas. A primeira amostra foi colocada em estufa a $50{ }^{\circ} \mathrm{C}$, até peso constante. À segunda amostra foram adicionados $3 \mathrm{ml}$ de água. A terceira amostra foi adicionada $3 \mathrm{ml}$ de álcool etílico $99 \%$ (Atlanta ${ }^{\circledR}$ Brasil), a quarta amostra utilizou-se o vegetal in natura e a quinta amostra foi $3 \mathrm{ml}$ de extrato de alho concentrado natural, Garlic Guard®.

O material vegetal foi acondicionado em uma Placa de Petri de $5 \mathrm{~cm}$, no quadrante quatro e um recipiente com água em cada quadrante próximo ao centro. A observação do quadrante em que se localizava cada animal foi realizada de 15 em 15 minutos durante duas horas, no período de maior atividade da espécie (06 às 08 horas), segundo Silva e Pelli, 2020.

A probabilidade de localização da $N$. cinerea nos quadrantes seria o mesmo caso não existisse repelência. Dessa forma foi utilizado o teste do $\chi^{2}$ (Chi-quadrado) para verificar a probabilidade do comportamento observado ser ao acaso ou não.

Como havia três quadrantes sem possível agente ativo e um com agente ativo, em $1 / 4$ ou $25 \%$ das observações, o animal deveria estar no quadrante com agente ativo, e em $3 / 4$ ou $75 \%$ das observações deveria estar nos outros três quadrantes. Foi considerado como significativo quando o valor de " $p$ " fosse igual ou inferior a $5 \%$.

\section{RESUlTADOS E DisCUSSÃo}

Diversos estudos descrevem ações próprias do alho nos organismos humano e animal, como medidas protetivas de doenças cardiovasculares (ERNST, 1987) e atividade repelente da diferentes organismos (SARTO, 1996). Quando colocado as formas de apresentação do Allium sativum, a maior diferença significativa foi observada para o extrato aquoso com valores de $\mathrm{p}$ entre $2,5 \%$ e $1 \%$. Estudos semelhantes que utilizaram o extrato aquoso do alho afirmam que o desempenho pode estar relacionado à provável presença da alicina (JUÁREZ-SEGOVIA et al, 2019). A alicina é o principal constituinte responsável pelas atribuições repelentes do alho (PARRA, 2011).

Entretanto, o extrato de alcoólico, o vegetal a seco, in natura e o óleo Guarlic obteve-se repelência no valor de p entre 5 e $10 \%$, o que pode estar associada à hipótese de que a constituinte 
alicina não esteja bioativo nestas formas, ou que não seja eficaz na repelência das baratas, pois estudos utilizando o extrato alcoólico do alho mostraram ser eficazes (OTA et al., 2010; COSTA et al., 2017).

Outros estudos semelhantes utilizando cercaria, uma larva de um parasita trematódeo, mostram a eficácia do óleo de Allium sativum (WAN et al.,2017; MUY-RANGEL et al.,2017; BOOYENS et al., 2013)

O alho tem sido eficaz no controle de endo e ectoparasitos, no caso de bovinos, sendo repelentes a insetos como a mosca-do-chifre (Haematobia irritans), carrapato (Boophilus microplus), e berne (Dermatobia hominis). Seu efeito repelente age após a ingestão, pois o produto libera odor característico tanto pelo suor como nas fezes, inibindo a reprodução das moscas (SARTO, 1997).

Tabela 1. Valor observado de p, pelo Teste do Qui-quadrado, para os experimentos comportamentais de Nauphoeta cinerea na presença de diferentes formas de apresentação e partes de Allium sativum.

\begin{tabular}{cc}
\hline $\begin{array}{c}\text { Forma de apresentação } / \\
\text { Parte da planta }\end{array}$ & Folha \\
\hline Extrato aquoso & $1 \%<\mathrm{p}<2,5 \% *$ \\
\hline Extrato alcoólico & $5 \%<\mathrm{p}<10 \%$ \\
\hline Planta seca & $5 \%<\mathrm{p}<10 \%$ \\
\hline in natura & $5 \%<\mathrm{p}<10 \%$ \\
\hline Óleo (tricoma foliar) & $5 \%<\mathrm{p}<10 \%$ \\
\hline
\end{tabular}

* valor considerado estatisticamente significativo, quando a probabilidade de o evento ser ao acaso for inferior a $5 \%$.

\section{CONSIDERAÇÕES FINAIS}

Os resultados deste estudo indicam que os extratos vegetais de Allium sativum possuem a capacidade de alterar o comportamento de $N$. cinerea e deve ser explorado já que pode ser uma alternativa aos inseticidas químicos no controle de populações de baratas. Entretanto a forma como o vegetal é preparado determina a eficácia ou não da planta como repelente, o uso de extratos vegetais é crescente e existem inúmeras possibilidades a serem pesquisadas, já que esse composto é de baixo custo, fácil preparo e não prejudica o meio ambiente.

\section{REFERÊNCIAS}

ADIRANO-ANAYA, M. L.; MEJÍA-ORTIZ, J.; OVANDO-MEDINA, I.; ALBORES-FLORES, V.; SALVADOR-FIGUEROA, M. Efecto de extractos alcohólicos de ajo (Allium sativum) y clavo (Syzygium aromaticum) en el desarrollo de Mycosphaerella fijiensis Morelet. Revista mexicana de fitopatología, v.36, n.3, p.379-393, 2018. 
BORLINGHAUS, J.; ALBRECHT, F.; GRUHLKE, M.C.; NWACHUKWU, I.D.; SLUSARENKO, A.J. Allicin: chemistry and biological properties. Molecules, v.19, n.8, p.12591618, 2018.

BOOYENS, J.; LABUSCHAGNE, M.C.; THANTSHA, M.S. In Vitro Antibacterial Mechanism of Action of Crude Garlic (Allium sativum) Clove Extract on Selected Probiotic Bifidobacterium Species as Revealed by SEM, TEM, and SDS-PAGE Analysi. Probiotics \& Antimicro. Prot., v.6, p.82-87, 2013.

CAVALCANTI, V.P.; ARAÚJO, N.A.F.; SCHWANESTRADA, K.R.F.; PASQUAL, M.; DÓRIA, J. Athelia (Sclerotium) rolfsii in Allium sativum: potential biocontrol agents and their effects on plant metabolites. Anais da Academia Brasileira de Ciências, v.90, n.4, p.3949-3962, 2018.

COSTA, N.C.; JUNIOR, A.F.C.; RAMOS, A.C.C.; SOARES, L.P.; SCHEIDT, G.N. Atividade antimicrobiana e análise fitoquímica preliminar do extrato vegetal de alho no controle de fungos fitopatogênicos. Revista Verde de Agroecologia e Desenvolvimento Sustentável, v.12, n.1, p.161-166, 2017.

ERNST, E. Cardiovascular effects of garlic (Allium sativum): a review. Phamatherapeutica, v.5, p.83-89, 1987.

FONSECA, G.M.; PASSOS, T.C.; NINAHUAMAN, M.F.M.L.; CAROCI, A.S.; COSTA, L.S. Avaliação da atividade antimicrobiana do alho (Allium sativum Liliaceae) e de seu extrato aquoso. Revista Brasileira de Plantas Medicinais, v.16, n.3, p.679-684, 2014.

JUÁREZ-SEGOVIA, K.G.; DÍAZ-DARCÍA, E.J.; MÉNDEZ-LÓPEZ, M.D.; PINA-CANSECO, M.S.; PÉREZ-SANTIAGO, A.D.; SÁNCHEZ-MEDINA, Y.M.A. Efecto de extractos crudos de ajo (Allium sativum) sobre el desarrollo in vitro de Aspergillus parasiticus y Aspergillus niger. effect of garlic extracts (Allium sativum) on the development in vitro of Aspergillus parasiticus and Aspergillus niger. Polibotánica, v.47, p.99-11, 2019.

KAKUMANU, M; MARITZ, J; CARLTON, J.; SCHAL, C. Overlapping Community Compositions of Gut and Fecal Microbiomes in Lab-Reared and Field-Collected German Cockroaches. Applied and Environmental Microbiology, v.84, n.17, p.1-17, 2018.

KUSANO, M.; KOBAYASHI, M.; IIZUKA, Y.; FUKUSHIMA, A.; SAITO, K. Unbiased profiling of volatile organic compounds in the headspace of Allium plants using an in-tube extraction device. BMC Res Notes, v.9, n.1, p.133, 2016.

MELO, B.A.; OLIVEIRA, S.R.; LEITE, D.T.; BARRETO, C.F.; SILVA, H.S. Inseticidas botânicos no controle de pragas de produtos armazenados. Revista Verde, v.6, n.4, p.1-10, 2011.

MUY-RANGEL, M.D.; OSUNA-VALLE, J.R.; GARCÍA-ESTRADA, R.S.; SAN MARTÍNHERNÁNDEZ, C.; QUINTANA-OBREGÓN, E.A. In vitro antifungal activity of garlic essential oil (Allium sativum L.) against Alternaria tenuissima. Revista Mexicana de Fitopatología, v.36, n.1, p. 162-171, 2017. 
OLIVEIRA, D.C.R.; SOARES, E.K.B.; FERNANDES, H.R.; BRASIL, L.S.N.S. Elaboração e caracterização físico-química, microbiológica e sensorial de pasta de alho condimentada com jambú (Spilantes oleraceae L.) desidratado. Scientia Plena, v.10, n.1, p.011501, 2014.

OTA, C.C.C.; DA SILVA, D.V.G.; JACON, K.C.; BAURA, V.; NUNES, S. Avaliação da atividade antimicrobiana e anti-inflamatória do extrato hidroalcoólico do Allium sativum (alho). Ciência e Cultura, v.43, n.43, p. 37-49, 2010.

PARRA, C.L.C.; OLIVO, C.J.; AGNOLIN, C.A.; SANGIONI, L.A.; BUZATTI, A.; PIVOTO, F.L. 2011. Soluções de alho no controle de nematódeos gastrintestinais de bovinos jovens. Santa Maria, UFSM, Tese de Mestrado na área de Produção Animal/Bovinocultura de Leite, 52p.

SARTO, M. Ração à base de alho combate à mosca-do-chifre. Notícia Sebrae. Campo Grande, MS. v.4, n.9, p.4-5, 1997.

SILVA, A.C.B.; PELLI, A. Estado atual do conhecimento das baratas, Ordem Blattaria BURMEISTER, 1829. Rev. UNINGÁ Review, Maringá, v. 34, n. 2, p. 28-38, 2019.

SILVA, A.C.B.; PELLI, A. metodologia para criação de três espécies de Blattaria Burmeister, 1829: Nauphoeta cinerea (Olivier, 1789), Blaberus giganteus (Linnaeus, 1758) e

Gromphadorhina portentosa (Schaum, 1853). Acta Biologica Brasiliensia, v. 3, n. 1, p. 25960016, 2020

SILVA, A.C.B.; PELLI, A. Ciclo Circadiano para Nauphoeta cinerea (Olivier, 1789) (Blattodea, Blaberidae) em condições climatizadas de laboratório. Braz. J. of Develop., v. 6, n. 9, p.6543765444, 2020.

WANG, K.; WANG, P.; ZHANG, L. In vivo and in vitro activity of oil extract of garlic (Allium sativum Linnaeus) against Schistosoma japonicum cercariae. Revista da Sociedade Brasileira de Medicina Tropical, v.50, n.1, p.126-129, 2017. 